\title{
Der Bericht C. Ruggieris über die Druckerei der Propaganda Fide
}

\author{
Eine Dokumentation
}

Superintendent Costantino Ruggieri erlebte die Druckerei der Propaganda Fide in Rom auf dem Höbepunkt ibrer Tätigkeit. Sein interessanter Bericbt behandelt die Entstehungsgeschichte der Druckerei von ibrer Grïndung im Jabre 1626 bis zum Jabre 1759. Mit dem Abdruck wurde in Heft Nr. 111976 im italienischen Original und in deutscher Úbersetzung begonnen.

\section{Der Wortlaut des Dokuments (3. und letzte Folge)}

\section{\IX. Primi lor Missonarj}

I primi falsi Missionarj Danesi, che fondarono con del successo questa Missione, furono Bartolomeo Ziegenbalg di Missen, ed Aurigo Plutschau, i quali arrivarono colà il di 9. di Luglio dell'anno 1706. La storia de' progressi di questa falsa Missione si legge accuratamente descritta da Gio. Alberto Fabricio Luterano, e da esso sappiamo ancora, che il primo pensiero di questi suoi settarj fù quello di apprender le lingue comuni di que' Paesi, cioè la nativa degl'Indiani Tamulica, o sia Malabarica, e la Telugica. e poi la Portoghese, la quale è familiare ai medesimi per il lungo commercio avuto con questa nazione.

\section{(75v)}

$\mathbb{X}$. Aprono una Stamperia in Tranquebar, e libri stampati nella medesima

Tuttociò si fece da coloro, per aver agio di aprire una Stamperìa nella stessa Città di Tranquebar, ed in Madrast, e vi riuscirono ben presto, perchè abbiamo il nuovo Testamento in lingua malabarica stampato in due sesti, cioè in 4, ed in 8 Tranquebariae typis et impensis Missionis Danicae A. D. 1714.

La stessa data porta il vecchio Testamento stampato colà in varj anni, cioè 1713. 26. 26.
\IX. Ihre ersten Missionare.

Die ersten falschen dänischen Missionare, die mit Erfolg diese Mission gegründet hatten, waren Bartolomäus Ziegenbalg59 aus Meissen und Heinrich Pluitschau ${ }^{60}$, welche am 9. Juli des Jahres 1706 dort ankamen. Die Geschichte der Erfolge dieser falschen Mission liest man sehr sorgfältig vom Lutheraner Joh. Albert Fabrizius beschrieben und von ihm wissen wir auch, daß der erste $\mathrm{Ge}-$ danke seiner Anhänger war, die üblichen Sprachen dieser Länder zu erlernen, d. h. die Eingeborenensprache der Inder Tamil oder die malabrische und die telugische, und dann die portugiesische, welche ihnen vertraut war durch den langen Handel mit dieser Nation.

$\$ X$. Sie eröffnen eine Drudkerei in Tranquebar und Bücher, die in ihr gedruckt wurden.

Alles das taten sie, um die Möglichkeit zu haben, eine Drudkerei in der Stadt Tranquebar selbst und in Madras' ${ }^{61}$ zu eröffnen, und es gelang ihnen sehr schnell, denn wir haben das Neue Testament in malabarischer Sprache, gedrudkt in zwei Formaten, d.h. in $4^{\circ}$ und in $8^{\circ}$ Tranquebar Typis et impensis Missionis Danicae A. D. 1714. Dasselbe Datum trägt das Alte Testament, das dort in 
28. in quattro volumi in 4. Ed oltre di questi abbiamo i Catechismi Luterani di varie specie più e meno voluminosi, e questi sono nel numero di sei.

Oltre di questi libri stampati nell'idioma di quel paese, si leggono ancora gli altri in lingua Danese, e Portoghese, e sono il Pentateuco, Catechismi, Grammatica, ed il libro de Imitatione Christi, e tutti quanti portano la data di Tranquebar.

$\$ X I$. Stamperìa degl'Inglesi in Madrast, e libri publicati nella med.a

La stamperìa di Madrast è servita per i libri tradotti in lingua Telugica, della quale essi hanno formata un'eccellente Gramatica, ed un copiosissimo Lessico in cinque colonne, (76r) due delle quali contengono le voci Tamuliche, o siano Malabariche corrispondenti agl'Inglesi, la terza la pronuncia, la quarta le voci Telugiche, e la quinta la corrispondenza delle voci Latine, ed ha la data di Madrastae 1728.

$\$ X I I$. Nuova stampa di lingue Orientali fondata in Hala dai Luterani

Ma quì non finisce lo zelo de' Luterani per la propagazione del Vangelo. Si è trovato in Hala di Sassonia un particolare, chiamato Gio. Enrico Callemberg, il qle coll'ajuto dei zelanti della setta, ha fondata una magnifica stamperì di lingue Orientali a posta per la conversione degl'Infedeli; ed in questa si stampano continuamente libri Ebraici, Arabici, Siriaci, Malabarici, Malaici, e Telugici. In quest'anno ha terminata la stampa del nuovo Testamento tradotto in lingua Indostanica, e ne ha fatto correre per l'europa l'avviso con pubblicare un piccolo scritto intitolato Narratio de edito novo Testamento Indostanico Halae 1758. Da questo scritto sappiamo ancora che Beniamino Schultz Luterano (76v) autore di questa versione ha pubblicato XII scritti Tamulici, XV Telugici, due Portoghesi, ed altrettanti Inglesi. verschiedenen Jahren gedrudkt wurde, d.h. $1713, ' 26,26,28$, in vier Bänden in $4^{\circ}$.

Außer diesen haben wir auch die lutherischen Katechismen verschiedener Art, mehr oder weniger umfangreich und ihre Zahl beträgt sechs.

Außer diesen in der Sprache des Landes gedrudkten Büchern, haben vir noch andere auf dänisch, auf portugiesisch; es sind der Pentateuch, Katedismen, eine Grammatik und das Buch der Nachfolge Christi und alle haben als Ort Tranquebar.

\$XI. Die Drudkerei der Engländer in Madras und von ihr herausgegebene Bücher ${ }^{61}$.

Die Druckerei in Madras diente für die in die telugische Sprache übersetzten Bücher, in der sie eine erstklassige Grammatik geschrieben haben und ein sehr umfangreiches Lexikon in fünf Kolumnen (76r) von denen zwei die tamulischen Wörter anführen oder das dem englischen entsprechende malabarische Wort, die dritte die Aussprache, die vierte die telugischen Wörter, die fünfte die entsprechenden lateinischen Wörter, und es trägt das Datum Madrastae 1728.

$\$ X I I$. Neue Druckerei der orientalischen Sprachen in Halle von den Lutheranern gegründet.

Aber hier hört der Eifer der Lutheraner bei der Verbreitung des Evangeliums nicht auf. Es hat sich in Halle in Sachsen ein Privatmann gefunden mit Namen Heinrich Callemberg62, der mit der Hilfe der Eifrigen seiner Sekte eine Druckerei der orientalischen Sprachen eigens für die Bekehrung der Ungläubigen gründete und in dieser drudkte man unaufhörlich hebräische, arabische, syrische, malabarische, malayamische und telugische Bücher. In diesem Jahr hat sie den Drudk eines in hindustanischer Sprache übersetzten Neuen Testaments beendet, und hat das in ganz Europa durch die Veröffentlichung einer kleinen Schrift mit dem Titel: Narratio de edito novo Testamento Indostanico Halae 1758 bekanntmachen lassen. Aus dieser Schrift wissen wir auch, daß der Lutheraner Benjamin Schultze (76v) der Autor dieser Úbersetzung, 12 tamulische, 15 telugische, zwei portugiesische und genausoviel englische Schriften veröffentlicht hat ${ }^{08}$. 
Capitolo VI.

Piano della Stamperia di Propaganda in confronto di quelle de' Protestanti

Se fosse vivo Monsig.e Ingoli primo Segretario di questa augusta Congregazione, e potesse vedere le generosita dei Protestanti intorno alla stampa dei libri per ifettare il mondo dei loro errori, sono più che sicuro, che egli non perderebbe l'occasione di farne il confronto colla strettezza presente della nostra stamperia di Propaganda, quantunque per la vastita ed ampiezza non abbia l'uguale in tutta l'Europa.

\section{\$I. Ricchezza della stamperìa della S. C.}

Chi meglio di noi potrebbe confondere questi falsi Missionarj, giacchè per la magnanimità dei Sommi Pontefici, e per la liberalità de' Principi ci troviamo una stamperìa fornita di 27 varie lingue, la maggior parte delle quali hanno le loro scale, le quali (77r) portano di una stessa lingua abbiamo più Alfabeti, come a cagion d'esempio dell'Arabo ne abbiamo di quattro specie, cioè Silvio, Antico, Garamone, e Garamoncino. Lo stesso dell'Armeno, del Siriaco, dell'Illirico e molto più del Latino, e del Greco, ed eccone il catalogo.

\section{Abissina}

2. Arabica

3. Armena

4. Bulgara

5. Bracmana

6. Caldaica estranghela

7. Caldaica Nestoriana

8. Coffta

9. Giorgiano letterale

10. Giorgiano volgare

11. Greca

12. Ibernese

13. Illirica di S. Girolamo

14. Illirica di S. Cirillo

15. Indiana

16. Latina

17. Moscovita

18. Persiana Ecclesiastica

19. Persiana volgare

20. Rutena

21. Serviana
Sechstes Kapitel

Plan der Propagandadrudserei

im Vergleich zu der der Protestanten.

Wenn Msgr. Ingoli, erster Sekretär dieser ehrwürdigen Kongregation am Leben wäre und die Großzügigkeit der Protestanten, um beim Druck von Büchern die Welt mit ihren Irrtümern zu infizieren, sehen könnte, bin ich mehr als sicher, daß er die Gelegenheit $\mathrm{zu}$ einem Vergleich mit der augenblicklichen Enge unserer Propagandadruckerei nicht verlieren würde, obschon es an Weite und Umfang keine ihr gleich in ganz Europa gibt.

\section{\$I. Reichtum der Drudkerei der H1. Kon- gregation.}

Wer besser als wir könnte diese falschen Missionare beschämen, da wir, durch die Hochherzigkeit der Päpste und die Freigebigkeit der Fürsten, eine Druckerei vorfinden, die mit 27 verschiedenen Sprachen ausgestattet ist, von denen die meisten ihre Skala haben, welche in (77r) derselben Sprache mehrere Alphabete haben, wie wir zum Beispiel auf arabisch vier Arten haben, d. h. Silvio, Antico, Garamone und Garamoncino. Dasselbe (gilt) vom Armenischen, vom Syrischen, vom Illyrischen und viel mehr vom Lateinischen und vom Griechischen, und hier ist der Katalog:

1. Abessinisch

2. Arabisch

3. Armenisch

4. Bulgarisch

5. Brahmanisch

6. Chaldäisch Stranguella

7. Chaldäisch Nestorianisch

8. Coptisch

9. Georgisch literarisch

10. Georgisch gewöhnlich

11. Griechisch

12. Irisch

13. Illyrisch von $\mathrm{Hl}$. Hyronimus

14. Illyrisch von Hl. Kyrill

15. Indisch

16. Lateinisch

17. Moskowitisch

18. Persianisch, kirchlich

19. Persianisch, gewöhnlich

20. Ruthenisch

(77v)

21. Serbisch 
22. Ebraica

23. Rabbinica

24. Tedesca

25. Samaritana

26. Siriaca

27. Tibetana

\section{\$I. Polzoni e Madri dei caratteri}

Nè questa strepitosa serie di caratteri fusi, che abbiamo nella stamperia, il solo peso de i quali passa le 40.000 libre è sterile per sè medesima; onde in caso, che col lungo uso si consumassero $i$ presenti, non ci fosse più modo di rifondergli in avvenire, perchè la Sagra Cong.ne è fornita di tutte quante le Madri dei medesimi toltene alcune poche scale Latine Siriache e Greche, ed in gran parte ancora de' Polzoni, onde si possano fondere di nuovo, e rifondere ad ogni occorrenza.

\section{\$III. Valore dei medesimi descritto da Monsig.e Ingoli}

Cosa importi una tal serie di Madri e di Polzoni, che ora sono distribuiti in 120 cassette, non si può meglio esprimere che colle parole stesse di Monsig.e Ingoli, estratte dalla sua prima relazione, ove descrive a minuto il grand'apparecchio fattosi per la stamperia, per muovere (78r) appunto lo zelo degli E.mi a non consegnarla in mano di uno stampatore mecanico - Si diede principio, dice egli, a detta fattura, e si finì; ed arrivato il numero de' Polzoni, e Madri sino a 23 diverse lingue le più universali, TESORO, che non si potrebbee più fare, perchè Paolini non vedo molto lume, ed il Tedesco anch'egli non sarà più bono, perchè nel fare detti Polzoni con tanti punti, e linee sottili vi vuole una vista esquisitissima, e gli occhiali non servono, che fanno alterar sempre le misure. Or pensi la $\mathrm{S}$. Cong.ne si è spediente consegnare questo TESORO ad uno stampatore, che non gl'intende, e se dovesse si conservaranno, come si fa adesso, con gran custodia; perchè se quelli della Vaticana sono diventati inutili per essersene perduti alcuni, e pur si mette sempre alla cura della Vaticana persona di valore, e di sapere, si puó considerare ciò, dhe succederà a quelli della Sac. Cong.ne massimamente, che morendo lo stampatore, Dio sa, ove capiteranno, se vi
22. Hebräisch
23. Rabbinisch
24. Deutsch
25. Samaritanisch
26. Syrisch
27. Tibetanisch

\section{Typen und Matrizen der Lettern.}

Diese erstaunliche Serie von gegossenen Lettern, die wir in der Drudkerei haben, deren Gewicht schon die 40.000 Pfund übersteigt, ist auch nicht unergiebig für sich selbst, denn im Fall, daß sich die augenblicklichen durch den langen Gebrauch abnutzen und keine Möglichkeit besteht, sie in Zukunft wieder einzuschmelzen, besitzt die Hl. Kongregation sämtliche Matrizen derselben, außer einigen wenigen lateinischen, syrischen und griechischen Maßeinheiten, und einen großen Teil der Typen, womit man sie schmelzen und wiedereinschmelzen kann bei jedem Bedarfsfall.

III. Wert derselben von Msgr. Ingoli beschrieben.

Was eine solche Serie von Matrizen und Typen bedeutet, die jetzt auf 120 Kassetten verteilt sind, kann man nicht besser ausdrücken, als mit denselben Worten von Msgr. Ingoli, entnommen aus seinem ersten Bericht, in dem er aufs genaueste die großen Vorbereitungsarbeiten beschreibt, die für die Drukkerei gemacht wurden, um eben den Eifer der Eminenzen zu bewegen (78r), sie nicht in die Hände eines mechanischen Druckers zu übergeben ${ }^{64}$ : „Man begann“, sagte er, „besagte Ausführung und beendete sie, und man ist bei der Zahl von Typen und Matrizen bis auf 23 der verschiedensten und allgemeinsten Sprachen gekommen, ein Schatz, den man nicht mehr schaffen könnte, denn Paolini sieht nicht mehr gut, und auch der Deutsche wird nicht mehr tauglich sein, denn beim Herstellen besagter Typen mit so viel Punkten und feinen Linien braucht man eine ausgezeichnete Sehkraft, und die Brille nutzt nichts, denn sie entstellt immer die Maße. Jetzt denkt die $\mathrm{Hl}$. Kongregation, ob es angebracht sei, diesen Schatz einem Drucker zu übergeben, der nichts davon versteht, und wenn man müßte, würde man sie unter guter Aufsicht aufbewahren, wie man es jetzt macht; denn wenn jene der Vatikana unnütz wurden, dann deshalb, weil einige verloren 
sarà Ministro della S.C. (78v) che li possa riconoscere, e ripigliare dagli Eredi.

E però da notarsi, che la mancanza de' Polzoni, e delle Madri Vaticane fù un artificio de' custodi di que' tempi, che erano certi Rinaldi, i quali parte per la lusinga, che essi avevano, dhe Urbano VIII. rimetesse in piedi la stamperìa Vaticana, disfatta da Paolo V., e parte per le solite gelosie, gli nascosero, e non li vollero dar tutti alla Sagra Cong.ne con tutto l'ordine del Papa; onde ne nacque, che nelle tre lingue Araba, Siriaca, ed Armena, parte sono in Propaganda, e parte si conservano ancora in Vaticana, con l'alfabeto Latino detto Canone Grosso, e sua Musica corrispondente, in otto cassette ben grandi, e quattro scatole.

\section{$\$ I V$. Stamperie dei Protestanti più ricche di quella della Sag. Cg.ne di quattro lingue}

Il fondamento adunque della stamperia della $\mathrm{S}$. Cong.ne non può essere nè più̀ magnifico, nè più durevole. Eppure con tutta questa gran serie di lingue, delle quali ella è fornita, tanto i Protestanti (79r) fanno più comparsa di noi, non già perchè essi abbino una stamperia più vasta della nostra, ma per le quattro sole lingue dell'Indie, cioè la Malaica, la Telugica, la Malabarica, o sia Tamulica, e l'Indostanica.

\section{$\$ \mathrm{~V}$. Ma con poco si superano}

$\mathrm{Ma}$ questo non dे un male irrimediabile, perchè se essi hanno saputo far intagliare $i$ Polzoni di que' caratteri, lo possiamo far ancor noi, quando avrem visti $i$ loro Alfabeti: nè la spesa è cosa, che debba porre spavento, perchè ben anche tutte queste quattro lingue portassero un migliajo di Polzoni, il the jo non credo, perchè l'Indostanica si serve dei caratteri Arabici, la spesa con tutte le Madri battute e aggiustate non oltrepasserebbe i 500 scudi incirca portarebbe il gettito dei caratteri. gingen. Und dennoch vertraut man die Vatikana immer Leuten von Wert und Wissen an, und man muß wissen und kann erwägen, was vor allem mit jenen der Hl. Kongregation passieren wird, wenn der Drudker stirbt; Gott weiß, wo sie dann hinkommen, ob dort ein Angestellter der $\mathrm{HI}$. Kongregation (78v) sein wird, der sie wiedererkennen und von den Erben zurückholen kann."

Es ist jedoch zu bemerken, $\mathrm{da} ß$ das Fehlen der Typen und der vatikanischen Matrizen ein Kunstgriff der Wädhter jener Zeit war, der gewissen Rinaldi, die sie zum Teil der Hoffnung wegen, die sie hatten, daß Urban VIII. die vatikanische Druckerei wieder aufbaute, die Paul V. aufgelöst hat, und zum Teil wegen der üblichen Eifersucht versteckten und sie wollten sie trotz des Befehls des Papstes nicht alle der Hl. Kongregation geben, so kam es, daß in den drei Sprachen Arabisch, Syrisch und Armenisch ein Teil in der Propaganda ist und ein Teil noch in der Vatikana aufgehoben wird, sowie das lateinische Alphabet, Großer Kanon genannt und seine entsprechende Musik in adht großen Kästen und in vier Schachteln.

\section{$\$ I V$. Um vier Sprachen reichere Drudkereien der Protestanten als der Hl. Kongrega- tion.}

Das Fundament der Drudkerei der Hl. Kongregation kann also weder großartiger noch dauerhafter sein. Trotz dieser großen Anzahl von Sprachen, mit denen sie ausgestattet ist, treten die Protestanten (79r) mehr als wir in Erscheinung, nicht weil sie eine größere Drudkerei als wir haben, sondern nur wegen der vier Sprachen von Indien, d. h. Malayalam, Telugisch, Malabarisch oder Tamil und die hindustanische.

\section{$\$ \mathrm{~V}$. Aber mit wenig überholt man sie.}

Aber dieses ist kein unabstellbares Ubel, denn, wenn sie es verstanden, Typen mit diesen Lettern zu gravieren, können wir das auch noch machen, wenn wir ihre Alphabete gesehen haben. Weder die Ausgabe ist eine Sache, die uns Erschrecken verursachen sollte, denn obwohl alle diese Sprachen etwa tausend Typen ausmachen, was ich nicht glaube, denn das Hindustanische bedient sich der arabischen Lettern; die Ausgabe mit allen geschlagenen und reparierten Matrizen würde 500 Scudi nicht überschreiten und ungefähr 
§I. Massima antica della S.C. di far sempre incidere gli Alfabeti di tutte lingue

Mille scudi non debbano disanimare in oggi la Sagra Cong.ne, quando ne spese da principio 18 mila tutti in un colpo, ed allora, co(79v) me ognun sa, era povera, e meschina; e ciò non ostante aveano già ideato di spendere di vantaggio per arricchirla di nuovi alfabeti, se fosse occorso, a misura, che $i$ Missionarj avessero fatto acquisto di nuovi regni, ed in fatti già si trova, che essi posero mano ai caratteri Cinesi, e Giapponesi, come si vede anche in oggi da più centinaja di legni incisi coi caratteri di queste due lingue, che sono rimasti nella stamperia; ed jo $\mathrm{mi}$ figuro, che non proseguissero il lavoro, non già perchè temevano della spesa, ma perchè successero le note rivoluzioni in que' due vastissimi regni, per le quali ne furon cacciati i Cristiani.

\section{S VII. Necessità dei libri cattolici}

Mi pare, che il servigio di Dio dovesse richiedere da noi questa premura perchè que' Paesi sono inondati di libri Eretici, e que' poveri Popoli vedono in stampa i Catechismi Luterani, e Calvinisti, e non il Catechismo Romano, ed i libri Sagri si leggono per generosità degli Ereti- (80r) tici, e non della Chiesa Romana.

\$VIII. Di questi ne abbiamo, ma non alla stampa

Degli eccelenti libri Cattolici ne giranco colà alcuni, ma scritti a mano per opera de' Missionarj Francesi, e del numero, e qualità di essi Monsig.e d'Eucarpia quì presente ne può rendere buona testimonianza ad ognuno. $\mathrm{Ma}$ la stampa renderebbe molto più comune la lettura, ed il pascolo della verità Cattolica, e quei poveri Fedeli restarebbero premuniti all'insidie degli Eretici, i quali non risparmiano spesa per sedurli. La Chiesa Romana, come Madre, e Maestra di tutt'i Cattolici, renderebbe comuni a tutto l'Universo questi weitere 500 Scudi würde das Gießen der Lettern kosten.

\$VI. Alter Grundsatz der HI. Kongregation immer die Alphabete aller Sprachen gravieren zu lassen ${ }^{88}$.

Tausend Scudi dürften die Hl. Kongregation heute nicht entmutigen, wenn sie am Anfang 18 Tausend auf einen Schlag ausgab (79r), und damals war sie wie ein jeder weiß, arm und bedürftig und trotzdem hatte man schon daran gedacht, zu ihrem Vorteil Ausgaben zu machen, um sie mit neuen Alphabeten zu bereichern, wenn es nötig war, und in dem Maß, wie die Missionare neue Reiche eroberten und in der Tat traf es sich, $\mathrm{daß}$ sie Hand an chinesische und japanische Lettern legten, wie man auch heute von einigen hundert gravierten Hölzern in diesen beiden Sprachen sieht, die in der Druckerei geblieben sind. Und ich stelle mir vor, daß sic ihre Arbeit nicht fortgesetzt haben, nicht weil sie die Ausgaben fürchteten, sondern weil sidh die bekannten Revolutionen in diesen beiden so großen Reichen ereigneten, durch welche die Christen verjagt wurden.

$\$$ VII. Notwendigkeit von katholischen Büchern.

Mir scheint, daß der Dienst Gottes diese Bemühung verlangt, weil diese Länder mit häretischen Büchern überschwemmt sind, und diese Völker sehen den Druck der lutherischen und calvinistischen Katechismen und nicht den römischen Katechismus; und die Heiligen Bücher liest man dank der Großzügigkeit der Häretiker (80r) und nicht von der Römischen Kirche.

$\$$ VIII. Diese haben wir, aber nicht im Druck.

Es sind dort ausgezeichnete katholische Bücher im Umlauf, aber sie sind handgeschrieben von französischen Missionaren und von ihrer Anzahl und Qualität kann der hier anwesende Msgr. d'Eucarpia einem jeden gutes Zeugnis ablegen. Aber der Drudk würde die Lektüre viel allgemeiner machen, sowie die Weide der katholischen Wahrheit, und diese armen Gläubigen wären bewahrt vor den Ränken der Häretiker, welche keine Ausgaben scheuen, um sie zu verführen. Die Römische Kirche, als Mutter und Meisterin aller 
atti solenni del Magistero confidatogli da Cristo.

\section{Capitolo VII.}

Si dimostra la necessità di un sito più addattato per la Stamperia

$\mathrm{Ma}$ prima di pensare a nessuna di queste imprese, è necessario di porre (80v) la stamperia in istato di poter servire, e di essere utile alla Sagra Congregazione, dhe è lo stesso, che alla Religione medesima.

\section{$\$ I$. Pessima qualità del sito presente}

Il sito, dov'è presentemente collocata, è affatto indecente, ed angusto; ed una Stamperia, la quale non ha pari in Europa, è assai peggio situata di quello sia la Stamperia di S. Michele a Ripa, ove si stampa il Donato, ed il Fiore di virtù, ed i libri scolastici per uso de i ragazzi, che vanno a scuole basse.

\section{$\$$ II. Variazioni del sito della medisma ne' tempi passati}

Già dal principio si è posta la variazione del sito della medesima, e si sono riferiti $\mathrm{i}$ Decreti della Sagra Congregazione per la Fabbrica di un Edificio commodo, e decente per la medesima. Si sa per testimonio di Monsig.e Ingoli, dhe il Cardinale Antonio Barberino, detto S. Onofrio, voleva fabbricargli un vaso a posta acciò la Stamperìa avesse lume, e commodo di operare.

\$III. Il Card. Capponi lascia la sua Eredità a Propaganda, perchè si faccia un Edificio p. la Stamperia

La stessa idea ebbe ancora il celebre Cardinale Luigi Capponi (81r) Bibliotecario della Vaticana, ed uomo di grandissima riputatzione nel Sagro Collegio; poicchè nel suo testamento rogato il di 24. di Giugno dell'anno 1657 istituì Erede la S.C. di Propaganda, ad oggetto, ch'Ella fabbricasse di pianta un'Edificio a posta, per collocarvi la
Katholiken, würde dem ganzen Universum die feierlichen Urkunden des ihr von Christus anvertrauten Amtes allgemein (zugänglich) machen.

\section{Siebentes Kapitel \\ Notwendigkeit eines geeigneten Platzes für die Druckerei.}

Aber bevor man an soldhe Unternehmen denkt, ist es notwendig (80v), die Druckerei in den Zustand zu versetzen, damit sie der Hl. Kongregation, was dasselbe ist, wie der Religion selbst, dienen und ihr nützlich sein kann.

$\$$ I. Schlechte Beschaffenheit des augenblicklichen Platzes.

Der Platz, wo sie augenblicklich angelegt ist, ist durchaus unwürdig und eng; und eine Druckerei, die keine gleich in Europa hat, ist viel schlechter gelegen als es die Druckerei von St. Michael in Ripa ${ }^{\mathrm{A7}}$, in der man den Donato, Fiore di vertù und Schulbücher für Kinder, die in die Elementarschulen gehen, druckt.

\II. Veränderungen des Platzes derselben in den vergangenen Zeiten.

Schon am Anfang hat man sich (die Frage der) Veränderung des Sitzes derselben gestellt, und man hat die Dekrete der Hl. Kongregation für den Bau eines bequemen und würdigen Gebäudes für sie gegeben. Man weiß, durch das Zeugnis von Msgr. Ingoli, daß der Kardinal Antonio Barberini, S. Onofrio genannt, eigens ein Gebäude errichten wollte, damit die Druckerei Licht und Bequemlichkeit zum Arbeiten habe.

\$III. Kardinal Capponi hinterläßt seine Erbschaft der Propaganda, damit man ein Gebäude für die Druckerei baut.

Dieselbe Idee hatte auch der berühmte Kardinal Luigi Capponi, Bibliothekar der Vatikana (81r), und ein Mann von sehr großem Ruf im Heiligen Kolleg, denn in seinem Testament vom 24. Juni des Jahres 1657, setzte er als Erbe die Hl. Kongregation ${ }^{68}$ der Propaganda ein, mit der Absicht, daß sie eigens ein Gebäude nach Plan erbaue, um 
Stamperia con tutti i commodi necessarj, de i quali in que' tempi, come già si è visto, n'era affatto priva. Ed ecco le parole stesse di quell'insigne Porporato - In tutti gli altri miei beni immobili, mobili, e le moventi, ed in tutte le ragioni, et azioni in qualsivoglia modo a me spettanti lascio, e dichiaro Erede la S. C. di Propaganda Fide, con ordine però, che avendo la medesima Sagra Cong.ne di Propaganda una Stamperia copiosissima, ed insigne per la quantità, e varietà de' caratteri, che in quella si conservano, ma priva di quelle commodità, the sono necessarie per una pronta, e facile impressione de' libri, si faccia quanto pri- (81v) ma col parere di tre, o quattro principali stampatori, e col disegno dell'Architetto della medesima Congregazione alla presenza del Cardinal Prefetto una fabbrica con tutti i membri, e parti necessarie per una stamperìa nel più commodo luogo, che sarà giudicato in piena Congregazione de' Sig.ri Cardinali, fabbricandosi con gli effetti della mia Eredità, li quali insino al compimento di detta fabbrica non possino essere impiegati in altro uso, stimando jo la stampa de' libri che s'imprimano propria della S.C. uno de' mezzi più principali d'adoperi la detta Congregazione per mantenimento e propagazione della nostra $S$. Fede, perchè $i$ libri penetrano, dove non possono arrivare $i$ Missionarj.

Luigi Cardinale Capponi proprio il giorno di S. Gio. Battista 1657.

$\$$ IV. Nel sito presente Ella fù collocata per pura, e mera provisione

Si sa ancora da i decreti accenati poc'anzi, che il sito ov'ella è presentemente, gli fù assegnato da principio per pura, e mera provisione, e che Ella in vece di migliorare, ha peggiorato, perchè dal primo, e secondo piano, che ella occupava, è stata poi ristretta nel solo pianterreno in due miserabili stanze, oscure, ed anguste. L'oscurità delle medesime è visibile a tutti, e questa porta un grandissimo incommodo per quando si compone nelle lingue Orientali, $i$ caratteri delle quali essendo pieni di minutissimi punti, si richiede dort die Druckerei mit allen notwendigen Vorteilen anzulegen, die ihr in diesen Zeiten, wie wir schon sahen, durchaus fehlten. Und hier die Worte selbst des hervorragenden Purpurträgers: „Für alle meine anderen beweglichen und unbeweglichen Güter und alle Rechte und Aktien, die mir in irgendeiner Weise gehören, hinterlasse ich und erkläre als Erben die Hl. Kongregation der Propaganda Fide, jedoch mit dem Befehl, da dieselbe Hl. Kongregation der Propaganda eine reichhaltige Druckerei, die hervorragt wegen der Quantität und der Verschiedenheit der Lettern, die in ihr aufbewahrt werden, der aber jene bequemen Arbeitsmöglichkeiten feblen, die für einen schnellen und leichten Druck der Bücher notwendig sind, soll man sobald (81v) wie möglich, jedoch nach dem Rat von drei oder vier bedeutenden Druckern und nach der Zeichnung des Architekten derselben Kongregation, im Beisein des Kardinals Präfekten, einen Bau mit allen notwendigen Gliedern und Teilen für eine Drukkerei an dem bequemsten Platz, der von der vollen Kongregation der Herren Kardinäle für richtig gehalten wird, mit den Effekten meiner Erbschaft errichten, die bis zur Vollendung des besagten Baues nicht für anderen Gebrauch benutzt werden können, denn ich schätze den Druck der Bücher, die man gerade in der $\mathrm{Hl}$. Kongregation für die $\mathrm{Er}$ baltung und die Verbreitung unseres Heiligen Glaubens gebraucht, denn die Bücher gelangen dorthin, wo die Missionare nicht hinkommen.

Luigi Kardinal Capponi, genau am Tag des Heiligen Joh. des Täufers 1657.“

\$IV. Am augenblicklichen Platz wurde sie nur aus reiner Vorläufigkeit untergebracht.

Man weiß noch aus den vorhin erwähnten Dekreten, daß ihr der Platz, wo sie augenblicklich ist, von Anfang an nur aus reiner Vorläufigkeit zugewiesen wurde, und daß sie, statt sich zu verbessern, sich verschlechterte, weil sie aus dem ersten und zweiten Stockwerke, die sie belegte, dann auf nur zwei enge, dunkle und miserable Zimmer im Erdgeschoß eingeengt wurde. Die Dunkelheit derselben ist für alle sichtbar, und dies bringt eine Beschwerlichkeit mit sich, vor 
perciò un sito luminosissimo, perchè i compositori possino fare il loro dovere con esateeza, ed attenzione.

\section{$\int V$. Disordini che nascono dall'angustie pre- senti}

L'angustia poi non è niente meno visibile, perchè non v'è sito da stendere le casse de' caratteri per comporre.

\section{\VI. Miseria de' torchj}

Deve ancora riflettersi, che una stamperia di XXVII lingue, e di XL e più mila libre di caratteri (82v) fusi si trova presentemente in piedi, che due miserabili torchj vecchi, e logori, ed assai peggiori di quelli della Camerale, che lavoravano a tempesta, com'è visibile ad ognuno. Una stamperia di tanta grandezza ne richiederebbe almeno sei per poter lavorare ad un colpo più cose, e farle bene. Ne' primi tempi Ella ne ebbe quattro, poi fù ridotta a tre, ed ora a due; e di questo terzo non ne abbiamo altro, che la sola vite di metallo, anzi cattiva, e logora.

Se si dovesse per cagion d'esempio stampare un'altro tomo della Biblioteca insigne di Monsignore Assemani, ricca di tante lingue, per comporre i fogli della medesima, si richiederebbe nelle presenti angustie il doppio più di tempo di quello si ridiedesse, quando la stamperìa fosse collocata in sito spazioso, in oui si potessero collocare tutte al pari sopra i loro piedi, o siano cavalletti le casse de' copiosissimi (83r) caratteri necessarj per una tal opera, ed $i$ compositori non fosser costretti dalla miseria presente di levare di mano in mano dagli Armarj le casse pesantissime de' caratteri, dhe gli bisognano, e stenderle sopra de' cavalletti, e poi subito terminata la composizione riporle negli Armarj suddetti, per dar luogo alle altre casse de' caratteri, che si debbano porre in opera dopo de' primi. allem, wenn man in den orientalisdhen Sprachen setzt, deren Lettern voller winziger Punkte sind. Das erfordert einen sehr hellen Platz, damit die Setzer ihre Pflidit mit Genauigkeit und Aufmerksamkeit erfüllen können.

\section{$\$$ V. Unordnung entsteht aus der augen- blicklichen Enge.}

Die Enge wird durchaus nicht weniger sichtbar, weil kein Platz zum Ausbreiten der Kästen zum Setzen der Lettern vorhanden ist.

\section{$\$$ VI. Misere der Pressen}

Man muß noch überlegen, daß eine Druckerei mit XXVII Sprachen und mit XI und mehr tausend Pfund gegossener Lettern (82v) augenblicklich mit nur zwei alten, miserablen und abgenutzten Pressen, die viel schlechter sind als jene der Camerale, die stürmisch arbeiten, wie es für jeden sichtbar ist, der sich aufrecht erhält. Eine Druckerei von diesem Ausmaß würde mindestens sechs anfordern, um mit einem Mal mehrere Sachen bearbeiten zu können und sie gut $\mathrm{zu}$ machen. In den ersten Zeiten hatte sie vier, dann wurden sie auf drei vermindert und jetzt auf $z$ wei. Und von dieser dritten haben wir nichts anderes, als nur die Metallschraube, die eher schlecht und verbraucht ist.

Wenn man, zum Beispiel, einen anderen Band der hervorragenden Bibliothek von Msgr. Assemani, die reich an vielen Sprachen ist, drucken müßte, würde das Setzen der Blätter derselben bei der augenblicklichen Enge das Doppelte an Zeit erfordern als nötig wäre, wenn die Drudkerei in einem geräumigen Platz aufgestellt wäre, indem man alle Kästen der so reichhaltigen Lettern, die für solch ein Werk nötig sind, gleichmäßig aufstellte, so müßten die Kästen als Bödke der zahlreichen Lettern dienen (83r), und die Setzer wären nicht von der augenblicklichen Misere gezwungen, nach und nach die sehr schweren Kästen mit den Lettern aus den Schränken zu heben, die sie benötigen, auf den Bödken auszubreiten und sofort, nachdem der Satz beendet ist, sie wieder in die besagten Schränke zurückzulegen, um den anderen Kästen mit Lettern Platz zu machen, die man nach den ersten benutzen muß. 
$\$$ VII. Spesa assai tenue che si richiede per collocarla magnificamente

Il decreto di questa Sagra Congregazione dei 15. Febraro 1644 porta, che si dovesse fabbricarne di pianta, come si è detto, un'Edificio a posta con tutte le officine necessarie, per collocarvi decentemente la stamperia, ed il Cardinal Capponi lasciò alla Sagra Cong.ne una pingue eredita, perchè si fabricasse un'Edificio a posta per collocarvi la stamperia. Se il decreto fosse stato eseguito, e la volontà di quell'E.mo avesse avuto effetto, la spesa per una fabrica somiglian- (83v) te, sarebbe stata, senza dubbio, molto considerabile, trattandosi di una stamperia di tanta vastita, per la quale si richiede un grandissimo sito.

$\mathrm{Ma}$ in oggi trovandosi fabbricato con tanta magnificenza il Collegio, non v'è bisogno più di tanta spesa, potendosi con pochissima trovare turto il comodo di collocare decentemente questa grand'opera senza il minimo incommodo della Comunità del Collegio. E se ho da dire il vero, quand'anche si volesse fabbricare un sito a posta per la medesima, non si potrebbe farlo nè più bello, nè più comodo di questo.

Mi giova sperare, che gli E.mi Sig.ri Cardinali, i quali compongono questa Sagra Congregazione, ben persuasi anch'essi della verita incontrastabile, che la nostra S. Religione si propaga, e si mantiene egualm.te con la viva voce de' Missionarj; e con i libri, e the $i$ Missionarj senza libri, come diceva Monsig.e Ingoli non pos- (84r) sono farsi, $e$ senza stampare de $i$ libri dogmatici, e disseminarli, non possono fare gran progressi; e che $i$ libri penetrano, dove non possono arrivare i Missionarj, come disse ancora il Cardinale Capponi; perciò, mi giova sperare, come ho detto, che l'Eminenze loro daranno tutta la mano, perchè si collochi decentemente un'opera di tanta importanza, la quale senz'alcun dubbio, può chiamarsi il braccio destro della Chiesa Romana per distendere il suo Magistero fino ai più rimoti confini della terra.
\$VII. Sehr geringe Ausgaben, um sie herrlicher unterzubringen.

Das Dekret dieser H1. Kongregation vom 15. Februar $1644^{\text {ob }}$ sagt, daß man nach einem Plan, wie man erwähnte, ein eigenes Gebäude mit allen nötigen Werkstätten bauen müßte, um die Druckerei würdig unterzubringen und Kardinal Capponi ließ der Hl. Kongregation eine reiche Erbschaft, damit man eigens ein Gebäude baue, um die Druckerei darin unterzubringen. Wenn das Dekret ausgeführt worden wäre und der Wille jener Eminenz Ausführung gehabt hätte, wäre die Ausgabe für einen gleichen Bau (83v) ohne Zweifel sehr ansehnlich gewesen, da es sich um eine Druckerei von solcher Größe handelte, die einen sehr großen Platz verlangte.

Aber heutzutage, da man das Kolleg mit solcher Großzügigkeit erbaut vorfindet, ist solch eine Ausgabe nicht mehr nötig, denn mit sehr wenig kann man eine bequeme und würdige Unterbringung für dieses große Werk, und ohne die geringste Beschwerlichkeit für die Kommunität des Kollegs finden. Und wenn ich die Wahrheit sagen soll, selbst, wenn man eigens ein Gebäude für dieselbe erbauen wollte, könnte man es nicht schöner und bequemer als dieses errichten.

Es empfiehlt sich zu hoffen, daß die Eminenzen, die Herren Kardinäle, aus welchen sich diese $\mathrm{Hl}$. Kongregation zusammensetzt, wohl auch von der unbestreitbaren Wahrheit überzeugt sind, daß sich unsere Heilige Religion verbreitet und sich erhält durch die lebendige Stimme der Missionare und zugleich durch die Bücher, und daß die Missionare, ohne Bücher es nicht werden ${ }^{67}$ können, wie Msgr. Ingoli sagte (84r), und ohne daß sie dogmatische Bücher drucken und sie verbreiten keine großen Fortschritte machen können, und daß die Bücher dorthin gelangen, wo die Missionare nicht hinkommen können, wie doch der Kardinal Capponi sagte. Deswegen empfiehlt es sich zu hoffen, wie ich sagte, daß die Eminenzen alle ihre Hilfe geben, damit man ein Werk von solcher Wichtigkeit würdig unterbringt, welches man ohne Zweifel den rechten Arm der Römischen Kirche nennen kann, um ihr Lehramt auszudehnen bis an die entlegensten Grenzen der Erde. 


\section{Anmerkungen:}

59. Bartholomäus Ziegenbalg (1682-1719) war luther. Theologe und begründete 1706 die Mission in Tranquebar. Er übersetzte Luthers kleinen Katechismus in Tamil, das er in ausgezeichneter Weise beherrschte.

60. Heinrich Plütschau (1677-1746) landete mit Ziegenbalg in Indien, das er jedoch bereits 1711 verließ. Er betreute vor allem die portugiesische Gemeinde.

61. Madras war ein englisches Missionszentrum, das von der Society for Promoting Christian Knowledge unterstützt wurde. Auch dort wurde eine Druckerei errichtet.

62. Johann Heinrich Callenberg war Prof. in Halle. Er wollte Missionar werden und studierte die arabische, persische und türkische Sprache. 1728 gründete er das Institutum Judaicum, das Literatur verbreitete und Missionare vorbereitete und unterstützte. Er starb 1760. Vgl. K. S. Latourette: A History of the expansion of Christianity, vol. 3 (1939) 61.

63. Benjamin Schultze war 1719-1725 Leiter der Tranquebarmission: ging danach nach Madras und kehrte 1741 nach Deutschland zurück. Vgl. J. Richter: Indische Missionsgeschichte (1924) 119, 122, 179, 396. Ruggieri ließ sich von Schultze Bücher schenken, als dieser zu Besuch in Rom war. Vgl. G. C. Amaduzzi: Commentarius 74.

64. Vgl. dazu W. Henkel: Francesco Ingoli ... In: „Communicatio Socialis“ (1970) 165-166.

65. Luigi Capponi wurde in Florenz geboren. 1608 Kardinal und seit 1623 Mitglied der Propaganda Fide, die er zeitweise als Pro-Präfekt leitere. Er starb 1659. Vgl. J. Metzler: Die Kongregation in der zweiten Hälfte des 17. Jahrhunderts. In: J. Metzler: S. C. de Prop. Fide Memoria Rerum I/1 (1971) 250-251.

66. Acta, vol. 16 (1644-45) f. $17 \mathrm{v}-18 \mathrm{v}$.

67. Vgl. "Communicatio Socialis“ (1970) 67. 